\title{
Inability of PET/CT to identify a primary sinonasal inverted papilloma with squamous cell carcinoma in a patient with a submandibular lymph node metastasis: A case report
}

\author{
SI-CONG ZHANG ${ }^{1,2}$, LIN WEI $^{1}$, SHUI-HONG ZHOU ${ }^{1}$ and KUI ZHAO ${ }^{3}$ \\ ${ }^{1}$ Department of Otolaryngology, The First Affiliated Hospital, College of Medicine, Zhejiang University, Hangzhou, \\ Zhejiang 310003; ${ }^{2}$ Department of Otolaryngology, People's Hospital of Cixi City, Ningbo, Zhejiang 315300; \\ ${ }^{3}$ Department of Positron Emission Tomography/Computed Tomography Center, The First Affiliated Hospital, \\ College of Medicine, Zhejiang University, Hangzhou, Zhejiang 310003, P.R. China
}

Received July 3, 2014; Accepted April 17, 2015

DOI: $10.3892 / \mathrm{ol} .2015 .3328$

\begin{abstract}
The present study describes the first reported case of a sinonasal inverted papilloma (IP) with squamous cell carcinoma (SCC) that presented as a cancer of unknown primary in the English-language literature. In July 2010, a 66-year old male presented with a 6-month history of a painless, progressive mass in the right submandibular region. Physical examination revealed a 3x4-cm smooth, non-tender mass and subsequently, excision of the right submandibular gland was performed. Post-operative pathological examination indicated poorly-differentiated SCC of the submandibular lymph node. Positron-emission tomography/computed tomography (PET/CT) was unable to identify the primary tumour site in this case, and 14 months later the patient presented with nasal obstruction, leading to the diagnosis of right sinonasal IP with coexistent SCC. The patient received pre-operative radiotherapy, a right total maxillectomy and post-operative radiotherapy. However, the patient succumbed to a distant metastasis 37 months after the initial presentation. ${ }^{18} \mathrm{~F}$-fluorodeoxyglucose uptake on PET/CT may be not a reliable predictor of malignancy in sinonasal IPs. Therefore, we suggest emphasis of the use of multiple biopsies for suspected sinonasal IPs in order to improve diagnostic accuracy.
\end{abstract}

\section{Introduction}

A sinonasal inverted papilloma (IP) is a locally aggressive, benign neoplasm with a high rate of recurrence. Sinonasal IPs

Correspondence to: Professor Shui-Hong Zhou, Department of Otolaryngology, The First Affiliated Hospital, College of Medicine, Zhejiang University, 79 Qingchun Road, Hangzhou, Zhejiang 310003, P.R. China

E-mail: zhouyunzhoush@163.com

Key words: inverted papilloma, nasal cavity, nasal sinus, positron emission tomography/computed tomography account for $0.5-4 \%$ of all sinonasal tumours (1), and are associated with malignant transformation or concurrent invasive squamous cell carcinoma (SCC) in $\sim 9 \%$ of cases (2-4). The one, three and five-year survival rates of sinonasal IP are are 80,71 and $63 \%$, respectively (1). The five-year survival rate of patients with sinonasal SCC is $~ 53 \%$ (5). It is difficult to distinguish a benign sinonasal IP from an IP with SCC or other malignant transformations during a routine workup with magnetic resonance imaging (MRI) or computed tomography (CT), as the malignant transformation is always local. Even histological examination of biopsied tissue may not identify SCC residing within an IP (2,3). A previous study indicated that positron-emission tomography (PET) or PET/CT with ${ }^{18} \mathrm{~F}$-fluorodeoxyglucose (FDG) may aid in the detection of sinonasal IP malignancies (6). However, other studies have reported that FDG-PET/CT is unable to differentiate between a benign sinonasal IP and a malignancy $(6,7)$.

To the best of our knowledge, there are no reported cases of sinonasal IPs with SCC presenting as a cancer of unknown primary (CUP). In the majority of cases of CUP, PET/CT is able to detect the site of the primary tumour $(6,7)$. However, the efficacy of PET/CT for the detection of primary tumours in patients with cervical carcinoma metastases remains to be determined (6). In a previous study, PET/CT was not able to identify a primary tumour or the use of PET/CT did not confer an additional advantage in primary tumour detection in patients with CUP (7).

In the present study, a case of right sinonasal IP with poorly-differentiated SCC, which presented as a submandibular lymph node metastasis, is reported. Notably, a high FDG uptake level was not present in the right nasal cavity on PET/CT, and avid local FDG uptake in the right maxillary sinus did not indicate the presence of a coexistent malignancy. Written informed consent was obtained from the patient's family.

\section{Case report}

A 66-year-old male who presented with a 6-month history of a painless, progressive mass in the right submandibular region 

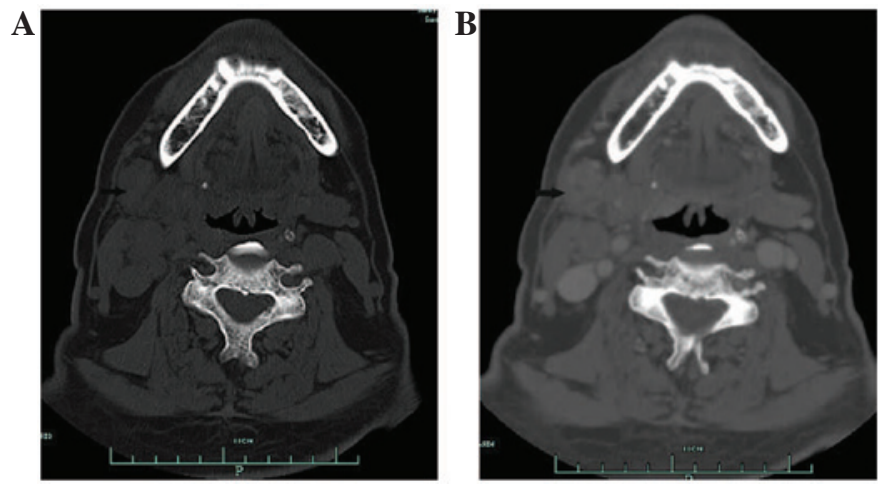

Figure 1. (A) Computed tomography scan of the submandibular region with a heterogeneous mass located near the right submandibular gland. (B) Contrast-enhanced imaging with peripheral, but not central lesion enhancement.

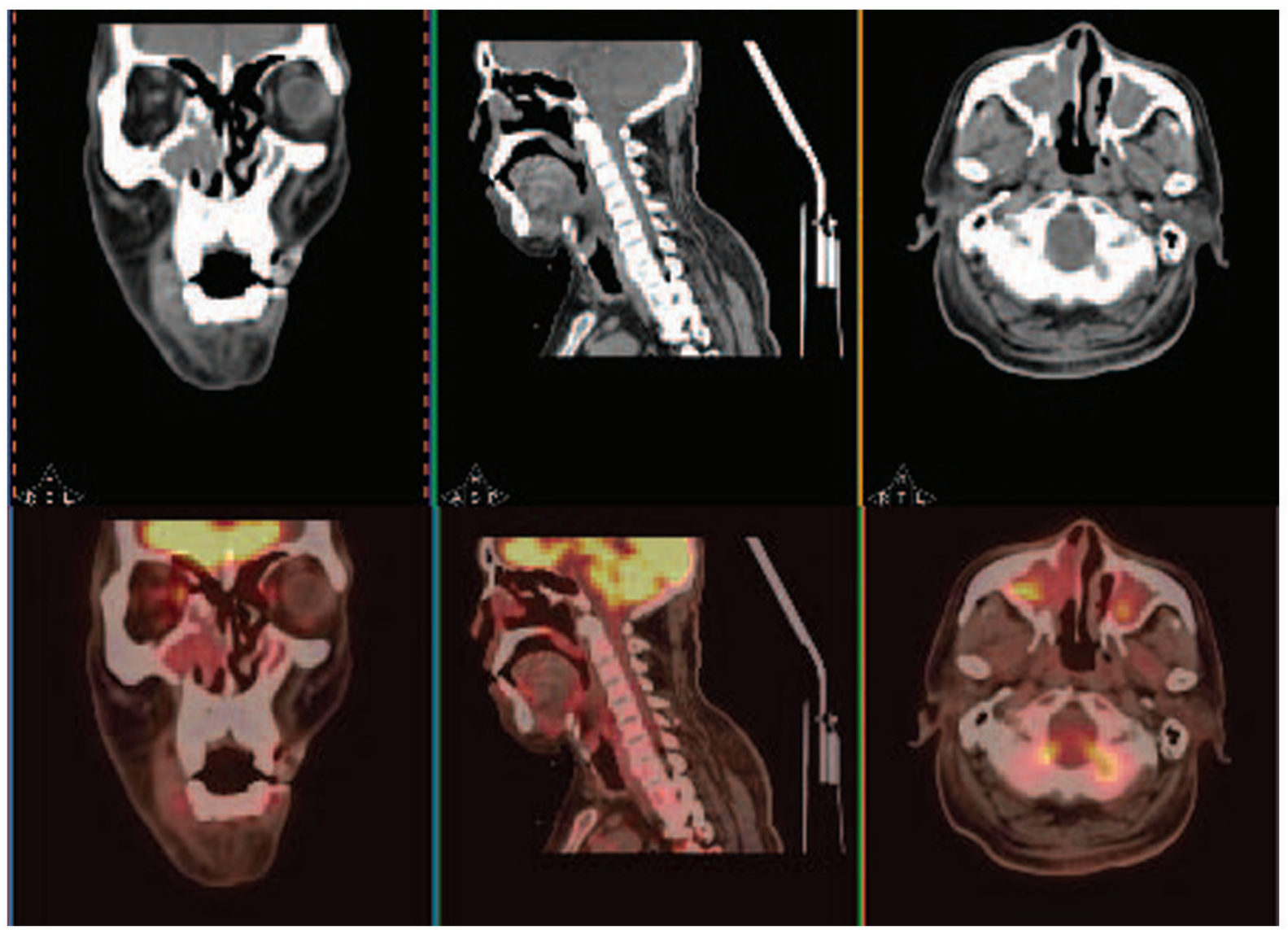

Figure 2. Positron emission tomography/computed tomography with local ${ }^{18} \mathrm{~F}$-fluorodeoxyglucose uptake in the bilateral maxillary sinuses (maximum standardised uptake value, 4.02), but no increased uptake in any other sites, including the right nasal cavity.

was admitted to the First Affiliated Hospital, College of Medicine (Hangzhou, China) in July 2010. The patient exhibited no dysphagia, dyspnoea, nasal obstruction, nasal bleeding or fever. A physical examination revealed a $3 \times 4-\mathrm{cm}$ smooth, non-tender mass in the submandibular gland region. The oral cavity and opening of the right submandibular duct were normal. The patient's past medical history included two enucleations of right nasal polyps, 14 and 8 years ago, respectively. A CT scan of the submandibular regions revealed a heterogeneous mass located outside of the right submandibular gland. Contrast-enhanced imaging demonstrated peripheral, but not central enhancement (Fig. 1). The initial diagnosis was of a pleomorphic adenoma or adenoid cystic carcinoma of the right submandibular gland. An excision of the right submandibular gland was subsequently performed. During surgery, the lesion was identified below the right submandibular gland, tightly adhered to the gland and the right marginal mandibular branch of the facial nerve. The lesion was completely excised and identified as a metastatic carcinoma by frozen section, which revealed large tumour cells with rich cytoplasm, a nested distribution and invasion of the lymph node tissue. The post-operative pathological analysis revealed atypical tumour cells, with a nested arrangement, and infiltration of the submandibular and cervical lymph nodes, confirming a diagnosis of poorly-differentiated SCC 
A

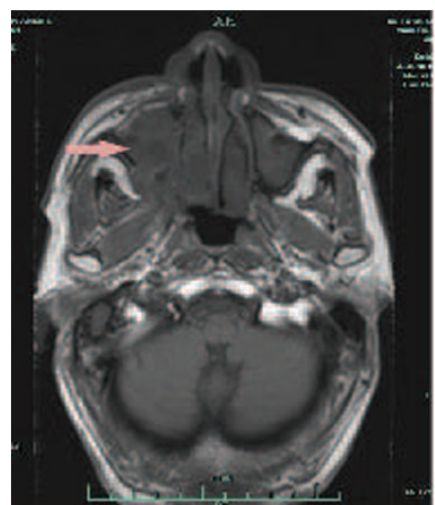

$\mathbf{B}$

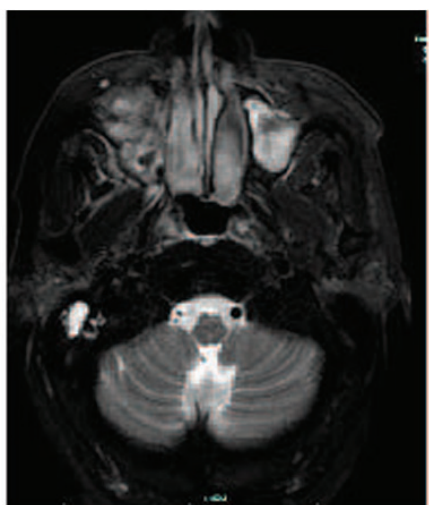

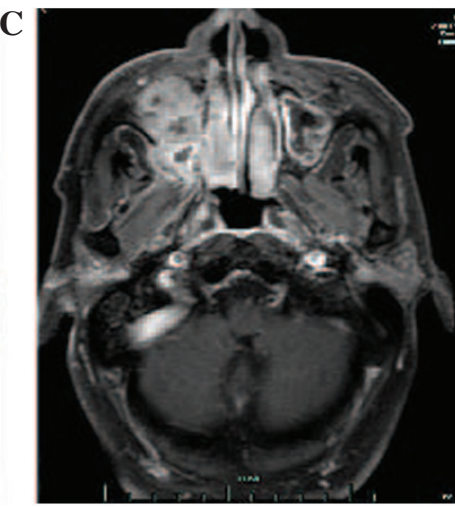

Figure 3. Magnetic resonance imaging of the nasal sinuses, with soft-tissue masses in the right nasal cavity and maxillary sinus 14 months after radiotherapy in right submandibular region. The medial, lateral and anterior walls of the right maxillary sinus had been destroyed by the tumour. Hyperintensity in (A) T2- and (B) T1-weighted imaging, with (C) marked enhancement following gadolinium-DTPA administration.
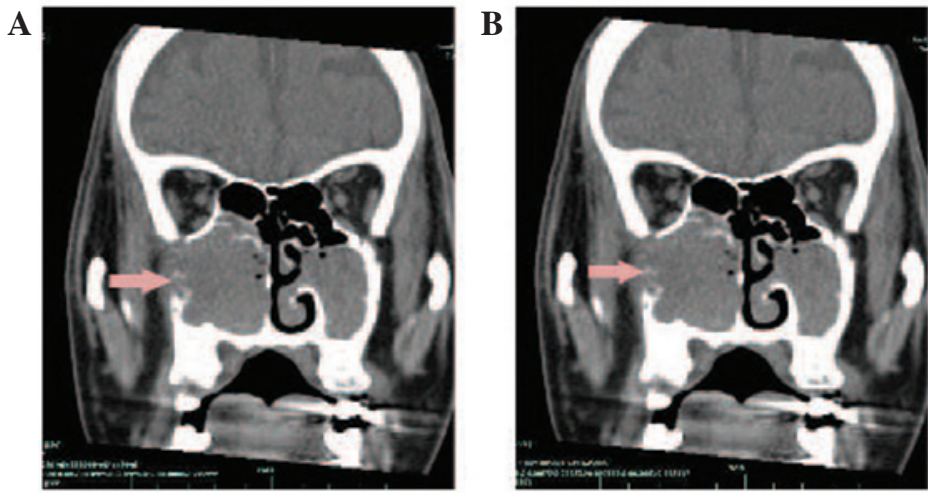

Figure 4. (A) A computed tomography (CT) scan with soft-tissue masses in the right maxillary sinus and nasal cavity 14 months after radiotherapy in the right submandibular region. The medial, lateral, anterior walls of the right maxillary sinus had been destroyed by the tumour, with a CT value of $28 \mathrm{HU}$. (B) Contrast-enhanced imaging with marked enhancement of the lesions at $812 \mathrm{HU}$. Arrows indicate the tumour.

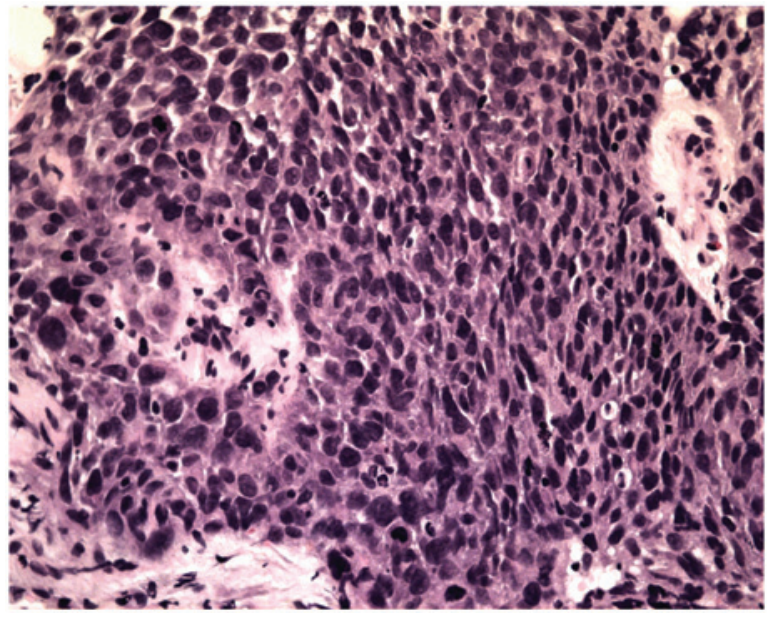

Figure 5. Biopsy of lesions in the right maxillary sinus and the right nasa cavity revealed inverted papilloma with squamous cell carcinoma (stain, hematoxylin and eosin; magnification, x100).

of the submandibular lymph node. A mass in the right nasal cavity was identified during nasal endoscopy, and the nasopharynx was normal. A biopsy of the right nasal cavity lesion revealed chronic inflammation. During PET/CT, local FDG uptake [maximum standardised uptake value $\left(\mathrm{SUV}_{\max }\right), 4.02$ ] was observed in the bilateral maxillary sinuses, but not in other sites in the body, including the right nasal cavity (Fig. 2). Therefore, the primary site was not identified. The patient received radiotherapy (60 Gy).

In September 2011, 14 months after the initial presentation, the patient presented with a 2-month history of a progressive right nasal obstruction. An MRI of the nasal sinuses revealed a soft-tissue mass in the right nasal cavity and maxillary sinus. The medial, lateral and anterior walls of the right maxillary sinus had been destroyed by the tumour. Hyperintensity was observed on T1- and T2-weighted imaging, and there was marked enhancement following gadolinium-DTPA administration (Fig. 3). A CT scan also showed soft-tissue masses within the right maxillary sinus and nasal cavity. The CT value was 28 HU. Contrast-enhanced imaging demonstrated markedly enhanced lesions at 812 HU (Fig. 4). A nasal endoscopy revealed that the right nasal cavity and right maxillary sinus were filled with oedematous, polypoid lesions. Biopsies of these lesions contained IP with SCC in the right maxillary sinus and nasal cavity (Fig. 5). The patient received pre-operative half-dose radiotherapy (30 Gy). The patient underwent a total right maxillectomy 1 month after the pre-operative radiotherapy, followed by another half-dose of radiotherapy (30 Gy) post-operatively. However, the patient succumbed to a brain metastasis in August 2013, 37 months after the initial presentation. 


\section{Discussion}

Currently no routine diagnostic methods are capable of differentiating between benign sinonasal IP and IP coexistent with carcinoma, since MRI and CT scans cannot differentiate between these diagnoses (3). Recently, a few studies found that PET or PET/CT was useful in detecting malignant transformation in sinonasal IPs. Shojaku et al (2007) reported that the $\mathrm{SUV}_{\max }$ of two patients with sinonasal IP and coexistent SCC were 8.9 and 20.9, which was higher than those of patients with IPs without SCC, which ranged from 4.9 to 7.3 (4). The study suggested that IPs with a high $\mathrm{SUV}_{\max }$ may be associated with malignancy even if the pre-operative biopsy is benign, and that a gross total excision of the IP and cancer should be performed in these cases, rather than removal of the IP alone (4). Allegra et al (8) found that FDG-PET/CT may aid in the detection of IP recurrence. The study showed that 21 patients exhibited an FDG uptake value that ranged from 4.5 to 8.1, and that an $\mathrm{SUV}_{\max }$ value of 8.1 was detected in a patient with a histological diagnosis of recurrent IP with SCC (8). However, Lee et al (2007) reported a case of benign IP in the maxillary sinus that had high SUV $\mathrm{max}_{\max }$ values $(9.0$ at $1 \mathrm{~h}$ and 18.1 at $2 \mathrm{~h}$ post-FDG injection), which were indistinguishable from those of malignant tumours (9). Cohen et al (2009) suggested that the degree of FDG uptake on PET/CT may be not a reliable predictor of malignancy in sinonasal IPs, as moderate to extremely high FDG uptake was also observed in benign sinonasal papillomas (2). Jeon et al (2009) reported that the SUV $\mathrm{Smax}_{\text {max }}$ o sinonasal IPs with coexistent SCC ranged from 13.3 to 31.9 (mean \pm standard deviation, 20.2 \pm 6.6 ), and that this range was higher than that of benign IPs (8.2 to 7.8; mean, 8.0) (3). The study also suggested that PET/CT cannot be used to reliably differentiation between benign IP and malignancy (3). The inability of PET/CT to differentiate between benign and malignant processes may be as FDG uptake is not specific to malignant processes, and other conditions, such as benign tumours or infectious processes, can also increase the rate of glycolysis $(2,3)$. Therefore, the role of FDG-PET/CT in the diagnosis of sinonasal IP and the differentiation of benign IPs from those with coexistent malignancies requires further investigation.

Cases of IP malignant transformation with cervical metastasis are rare (10). Only two cases of sinonasal IPs with SCC and cervical metastasis have previously been reported in the English-language literature $(10,11)$. Mathew et al reported a case of sinonasal IP with coexistent malignancy that progressed to the cervical lymph nodes in regions IIB and III (10). Mazlina et al reported a case of multicentric IP in the sinonasal region and middle ear in which the patient developed a cervical metastasis secondary to malignant transformation of the IP in the middle ear (11). Recently, Karam et al reported a case of sinonasal IP coexistent with high-grade esthesioneuroblastoma in which the patient developed metastases bilaterally in the cervical lymph nodes of regions I and II (12). To the best of our knowledge, sinonasal IP with coexistent SCC presenting as a CUP has not previously been reported. In the present case, the patient initially presented with enlarged cervical lymph nodes in region I. FDG-PET/CT revealed an $\mathrm{SUV}_{\max }$ of 4.02 in the bilateral maxillary sinuses, but increased FDG uptake was not observed in the right nasal cavity. Based on previous studies and our experience, the FDG-PET/CT findings in the present case did not suggest a coexistent malignancy in the nasal cavities or sinuses. Additionally, a biopsy of the right nasal cavity found inflammation. These findings indicate that biopsied tissue may not always identify SCC within a sinonasal IP, particularly if the SCC only resides in a small portion of the sinonasal IP (3). Thus, multiple biopsies should be conducted to improve diagnostic accuracy.

It has been reported that PET/CT aids in the detection of primary tumours in CUP cases (6,7). In our previous study, the sensitivity of FDG PET/CT in detecting primary tumours was $73.3 \%$ and the positive predictive value was $52.4 \%$ (6). These findings indicated that PET/CT has certain limitations in detecting the primary site of the CUP. Although several studies have demonstrated that PET/CT imaging may be useful in the evaluation of IPs with SCC, particularly group 2 and 3 lesions (13), the findings in the present case, as well as in other studies $(2,3,13)$, demonstrated that PET/CT does not reliably identify sinonasal IP with SCC. Therefore, we suggest that low FDG uptake by a sinonasal IP does not exclude the possibility of malignant transformation or coexistent malignancy, even in IPs with benign pre-operative biopsies.

In conclusion, sinonasal IPs with coexistent malignancy and cervical metastasis are rare. The present study describes the first reported case of a sinonasal IP with coexistent SCC that presented as a CUP. FDG uptake on PET/CT may be not a reliable predictor of malignancy in sinonasal IPs, and we suggest the use of multiple biopsies of suspected IPs in order to improve diagnostic accuracy.

\section{Acknowledgements}

This study was supported by the National Natural Science Foundation of China (grant no. 81172562).

\section{References}

1. Tanvetyanon T, Qin D, Padhya T, Kapoor R, McCaffrey J and Trotti A: Survival outcomes of squamous cell carcinoma arising from sinonasal inverted papilloma: Report of 6 cases with systematic review and pooled analysis. Am J Otolaryngol 30: 38-43, 2009.

2. Cohen EG, Baredes S, Zuckier LS, Mirani NM, Liu Y and Ghesani NV: 18F-FDG PET evaluation of sinonasal papilloma. AJR Am J Roentgenol 193: 214-217, 2009.

3. Jeon TY, Kim HJ, Choi JY, et al: 18F-FDG PET/CT findings of sinonasal inverted papilloma with or without coexistent malignancy: Comparison with MR imaging findings in eight patients. Neuroradiology 51: 265-271, 2009.

4. Shojaku H, Fujisaka M, Yasumura S, et al: Positron emission tomography for predicting malignancy of sinonasal inverted papilloma. Clin Nucl Med 32: 275-278, 2007.

5. Turner JH and Reh DD: Incidence and survival in patients with sinonasal cancer: A historical analysis of population-based data. Head Neck 34: 877-885, 2012.

6. Zhao K, Luo XM, Zhou SH, et al: ${ }^{18} \mathrm{~F}$-fluorodeoxyglucose positron emission tomography/computed tomography as an effective diagnostic workup in cervical metastasis of carcinoma from an unknown primary tumor. Cancer Biother Radiopharm 27: 685-693, 2012.

7. Park JS, Yim JJ, Kang WJ, et al: Detection of primary sites in unknown primary tumors using FDG-PET or FDG-PET/CT. BMC Res Notes 4: 56, 2011.

8. Allegra E, Lombardo N, Cascini G, La Boria A, Garozzo A and Tamburrini O: Possible role of 18FDG-PET/CT for the surveillance of sinonasal inverted papilloma. Clin Otolaryngol 35: 249-251, 2010. 
9. Lee KW, Kuo WR, Tsai CC, Chen YW, Chai CY, Su YC and Lin CS: Positive positron emission tomography/computed tomography in early inverted papilloma of the maxillary sinus. J Clin Oncol 25 : 4848-4850, 2007.

10. Mathew P and Idiculla JJ: Malignant sinonasal papilloma with neck metastasis: a rare report and literature review. Int J Oral Maxillofac Surg 41: 368-370, 2012.

11. Mazlina S, Shiraz MA, Hazim MY, Amran AR, Zulkarnaen AN and Wan Muhaizan WM: Sinonasal inverted papilloma with malignant transformation in the middle ear: a multicentric origin? J Laryngol Otol 120: 597-599, 2006.
12. Karam SD, Jay AK, Anyanwu C, Steehler MK, Davidson B, Debrito P and Harter KW: Pathologic collision of inverted papilloma with esthesioneuroblastoma. Front Oncol 4: 44, 2014.

13. Barnes L, Vervin RS and Gnepp DR: Diseases of the nose, paranasal sinuses, and nasopharynx. In: Surgical Pathology of the Head and Neck. Barnes L (ed). Vol 1. Marcel Dekker, New York, NY, 403-451, 1985. 